\title{
VACUUM-PUMP CONTROL SYSTEM USING PROGRAMMABLE LOGIC CONTROLLERS ON THE TCP/IP NETWORK FOR THE 2.5-GEV STORAGE RING
}

\author{
Noriichi KANAYA ${ }^{\#}$, Seiji ASAOKA and Hideki MAEZAWA \\ Photon Factory \\ High Energy Accelerator Research Organization (KEK) \\ 1-1, Oho, Tsukuba, 305-0801, Japan
}

\begin{abstract}
A vacuum-pump control system has been developed using programmable-logic controllers (PLC) for the 2.5$\mathrm{GeV}$ storage ring at the Photon Factory, High Energy Accelerator Research Organization (KEK). There are sixty-six titanium-getter vacuum pumps at the storage ring. Evacuation of gases in the storage ring is done by controlling the current in the titanium filaments in the vacuum pump (max 50A). A PLC has a TCP/IP network port, 16-bit-digital output ports connected to sixteen solid-state relays (SSR) for current control. The PLC can simultaneously control up to sixteen pumps. These vacuum pumps are connected to SSRs which chop the $\mathrm{AC}$ current so as to control the current in the pumps. To operate the pumps, the pump current must have a trapezoidal-shaped current form. The PLC is configured so that the current in the pumps has a trapezoidal form associated with pre-heating, evacuation, and coolingdown phases of the pump. PLCs are connected to a personal computer (PC) through the network. The PC can automatically control the PLCs by sending a set of commands through the TCP/IP network. The commands specify the duration of the current form. Upon receiving a command from a PC running under WindowsNT through the network, the PLC generates pulse-trains through the digital output ports to trigger the SSRs in association with the operating phases. The design of the vacuum-pump control system is discussed.
\end{abstract}

\section{INTRODUCTION}

There are twenty-two synchrotron-radiation beam lines at the $2.5-\mathrm{GeV}$ positron storage ring, Photon Factory at the High Energy Accelerator Research Organization (KEK). These beam lines feed synchrotron radiation to the experimental hall, where experiments, such as surface physics, x-ray lithography, microscopy and crystal

" Email: noriichi.kanaya@kek.jp structure analysis, are simultaneously carried out. These beam lines are simultaneously in operation, providing intense synchrotron-radiation beams. The pressures in the storage ring and the beam lines are maintained at an ultra-high vacuum (UHV) of less than $10^{-8} \mathrm{~Pa}$ so as to achieve a long beam lifetime, typically more than sixty hours. The beamline are controlled by the distributed control system [1].

There are sixty-six titanium-getter pumps for the rough evacuation of gases in the storage ring. The vacuum pumps are installed around the storage ring, and have to be periodically operated, depending upon the pressures in the storage ring. The evacuation of gases in the storage ring is done by controlling the current in the titanium filaments in the vacuum pump $(\max 50 \mathrm{~A})$. The control system for the titanium-getter pumps has been developed using Programmable Logic Controllers on the TCP/IP network at the Photon Factory.

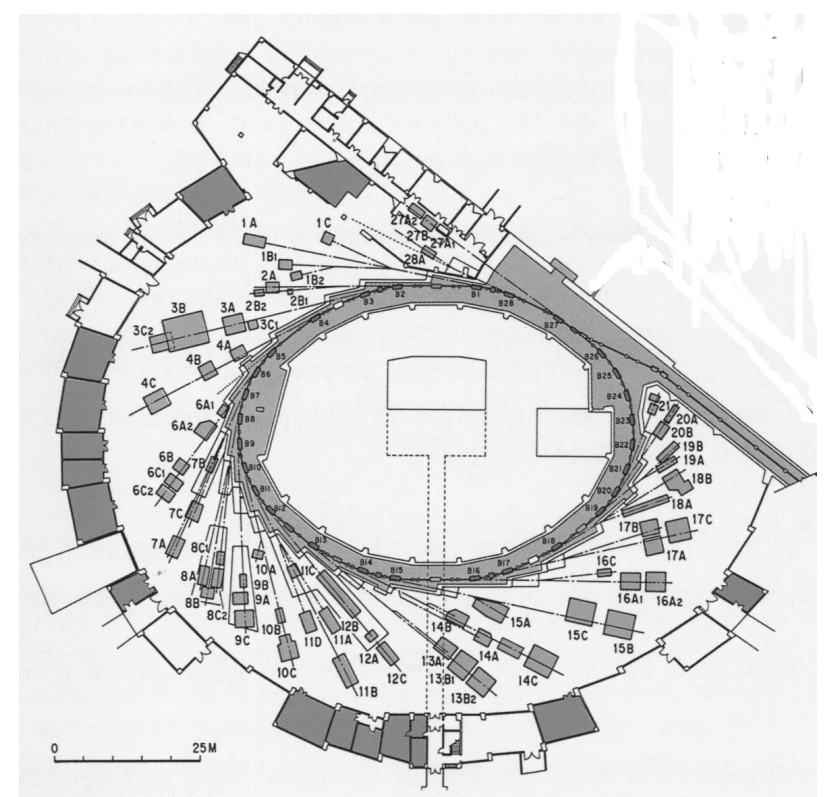

Fig.1 The 2.5-GeV storage ring and beamlines 
The titanium-getter pumps are installed at each beamline.

\section{SYSTEM CONFIGURATION}

Figure 2 shows the configuration of the system. A PLC has a 16-bit CPU (Intel 80386, 25MHz), a 2 MB DRAM, a TCP/IP network port and two sets of 16-bit-digital input/output ports connected to sixteen solid-state relays (SSR) for current control. The PLC can simultaneously control up to 16 pumps. These vacuum pumps are connected to the SSRs that chops the AC current so as to control the current in the pumps.

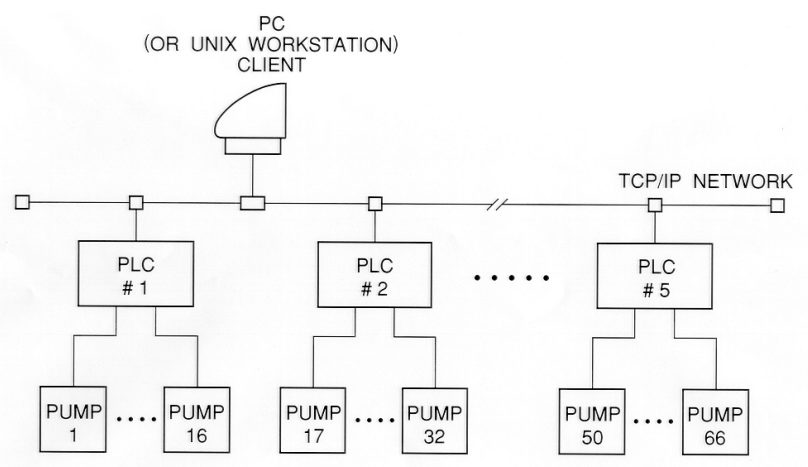

Fig.2 Configuration of the control system for titaniumgetter pumps.

To operate the pumps, the pump current must have a trapezoidal shape as shown in Fig.3. The PLC is configured so that the current in the pumps has a trapezoidal form associated with the warming-up phase, evacuation phase, and cooling-down phase of the pump. Usually thyristors are employed to control the AC current for high-power devices, allowing us to linearly control the electric power in the power devices. However, thyristors can generate chopping noise when slicing the AC current to control the power for the power devices. In order to avoid such noise generated by chopping the high AC current, the PLC employs SSRs that can chop the $\mathrm{AC}$ current $(50 \mathrm{~Hz})$ at the zero-crossing point, thus providing an arbitrary number of sinusoidal current waves to the filaments of the titanium-getter pump without much noise. The PLC controls the number of sinusoidal current wave forms ranging from 0 to 50 in a second for the titanium-getter pump. Thus, the PLC can control the average $\mathrm{AC}$ power consumed in the titaniumgetter pump, thus ensuring a trapezoidal-shaped current.

The PLCs are connected to a personal computer (PC) through the network. The connection between the PC and the PLC is established using the TCP/IP socket mechanism. The PC is a client and the PLCs are servers. The PLC has only a simple connection mechanism i.e. the "socket mechanism" rather than Remote Procedure
Call (RPC) that supports rich client-server mechanism. Thus, communication between the PLCs (servers) and the PC (client) is established using the socket

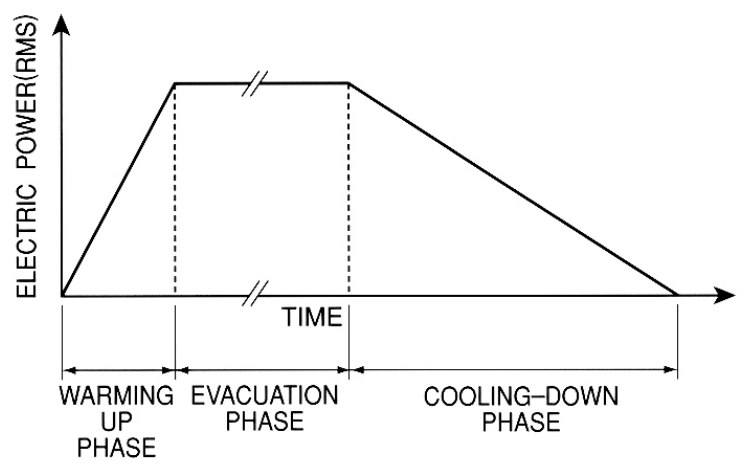

mechanism.

Fig.3 Trapezoidal-shaped current (RMS) in the titaniumgetter pump. The current in the pump must have a trapezoidal form associated with the warming-up phase, evacuation phase and cooling-down phase.

The PC can automatically control PLCs by sending a set of commands through the TCP/IP network. Control for the titanium-getter pumps is done by sending commands, for example, the execution command specifies the duration of the current form: the duration of warming-up phase, the duration of evacuation phase and the duration of cooling-down phase. The PLC listens to the TCP/IP port to receive a message or command from the PC through the network. Upon receiving a command from the PC running under WindowsNT through the network, the PLC generates pulse-trains through the digital output ports to trigger the SSRs in association with the operating phases, as shown in Fig.3. A command includes an ID number, which specifies the titanium-getter pump as well as the duration of the warming-up, evacuation, and cooling-down time in seconds. Upon receiving a command, the PLC initializes its timers associated with the command parameters, and starts triggering the gate of the SSR through the digital output port. Triggering the SSR chops the AC voltage that is fed to the titanium-getter pump, thus controlling the AC current in the filament of the titanium-getter pump. After setting up the timers, the PLC listens to the TCP/IP port again for the next command from the client.

In the case that the filament of the titanium-getter pump is burnt out, the PLC can automatically abort triggering the SSR. In order to avoid a high surge current when aborting, the PLC gradually reduces the number of triggering pulses for the SSR gate. This procedure can also be initiated by the PC by sending the abort command.

The man-machine interface on the PC was created using Java. Figure 4 shows a sample screen copy of the man-machine interface, which is in the development 
phase. The operator can flash all titanium-getter pumps, or individually flash arbitrate combinations of the pumps by clicking the radio button on the screen. The functionality of the client on the PC will be ported to a UNIX workstation.

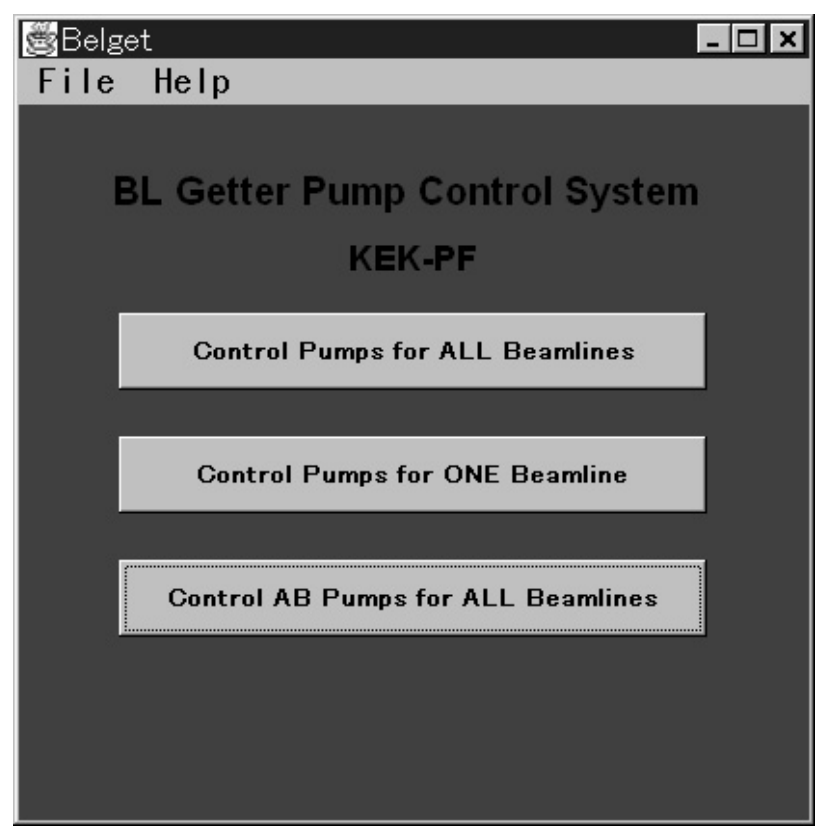

Fig.4 (a)

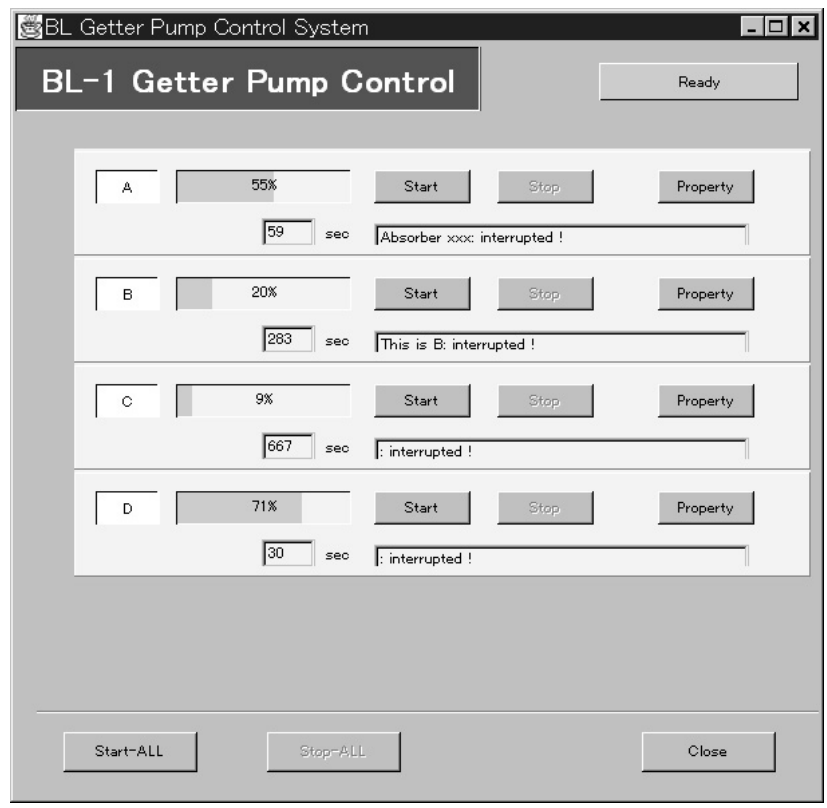

Fig.4 (b)
Fig.4 Sample screen copy of the man-machine interface of the client. (a) Main menu and (b) a sub-menu controlling four sets of titanium-getter pumps.

\section{CONCLUSION}

A vacuum-pump control system has been developed using programmable-logic controllers (PLC) for sixty-six titanium-getter vacuum pumps at the $2.5-\mathrm{GeV}$ storage ring at the Photon Factory. The PLC has sixteen solidstate relays (SSR) that can control the AC current to control the current in the pumps. The PLCs and a PC are connected in development phase, and will be installed for all titanium-getter pumps.

\section{ACKNOWLEDGEMENTS}

The authors wish to express their gratitude to the staff of the Photon Factory Light Source Division for operating the beam lines and the storage ring.

\section{REFERENCE}

[1] N.Kanaya, S.Sato, S.Asaoka, T.Koide and H.Maezawa, "Operational Experience with the Distributed Control System for Synchrotron Radiation Beam Lines at the Photon Factory", Particle Accelerators, Vol.29,pp.227-232,1990. 